\title{
NUMERICAL ANALYSIS ON THE INFLUENCE OF THE TWISTED BLADE ON THE AERODYNAMIC PERFORMANCE OF TURBINE
}

\author{
Jianguo Jin \\ Zhanzhou Wang \\ Lihua Cao \\ School of Energy and Power Engineering, Northeast Dianli University,Jilin,Jilin, China
}

\begin{abstract}
With the gradual increase of the thermal power unit capacity, the inlet steam parameters and flow of the turbine also increase gradually, which causes considerable secondary flow loss. Therefore, studying the causes and distribution of secondary flow loss within the level is of great significance to effectively improve the stage internal efficiency of turbine. Take high-pressure stage moving blade of a turbine as the research object, and adopt the ${ }^{k-\omega}$ SST model, the SIMPLEC algorithm to numerically simulate the formation and development process of leakage vortex between the tip clearance of the positive bending twisted blade and its effect on the secondary flow of cascade passage. Results show that relative to the conventional twisted blade, the scope of influence of leakage vortex which the steam flow formed near the suction surface of positive bending twisted blade and the disturbance to passage mainstream become smaller, and the increase of tip clearance has weakened the "C" type pressure gradient of suction surface of the positive bending twisted blade, increased the thickness of the boundary layer at both ends of blades and the loss of the blade end.
\end{abstract}

Keywords: Turbine, Twisted blade, Leakage vortex, Pressure gradient, Tip clearance.

\section{INTRODUCTION}

The bending of turbomachinery twisted blade includes positive bending and reverse bending. The included angle between pressure side of the blade and the superior and inferior end walls is obtuse angle, which is reverse bending, and acute angle is positive bending twisted blade. Positive bending twisted blade can make the steam flow in the blade surface, especially nearby the suction surface form "C" type pressure distribution of the positive radial pressure gradient and the negative radial pressure gradient of the blade tip. The "C" type pressure distribution will help to entrain low energy fluid at the superior and inferior end walls into the mainstream region of the central area, so as to effectively control the secondary flow of fluid in the end wall [1]. A large number of studies have shown that positive bending twisted blades adopted in the blade design can reduce the flow loss in the cascade passage and improve the performance and operational efficiency of the turbomachinery [2-3].

At present, bending technology of twisted blades has had abundant theoretical analysis and experimental research results. B.T. An, etc [4] studied the turbine cascade flow field under different conditions of bending, and pointed out the small angle positive bending design of blade can make the passage vortex of the end region strengthen, angle area separate and disappear, so as to achieve the effect of reducing the flow loss of the end and improving the aerodynamic performance of the turbine. Through the wind tunnel experimental study of high-pressure annular cascade, Z.M. Feng, etc [5] got appropriate coordination of rear load blade 
profile with the positive bending blade, which can further optimize the three-dimensional pressure field inside the cascade, and reduce the profile loss and secondary flow loss. The study of H.W. Lu, etc $[6,8]$ shows that passage vortex strength of positive bending cascade in the compressor increases, the wall vortex intensity weakens, and the structure of the passage vortex generates relatively complex changes. L. Chen, etc [7] studied the comprehensive influence of bending blades on the aerodynamic performance of the turbine, and proposed only if the interaction between blade rows be fully considered, can the best aerodynamic performance scheme of the turbine bending blade design be found.

Based on the computational fluid dynamics software Fluent, the article conducts a numerical simulation on the flow field of high-pressure stage positive bending twisted blades of a turbine with blade tip clearance, probes the formation and development process of tip clearance leakage flow and vortex of the positive bending twisted blades in high-pressure stage environment, and emphatically analyzes the influence of the tip leakage flow of positive bending twisted blades on the secondary flow of the cascade passage under different blade tip clearance.

\section{THE CALCULATION MODEL AND NUMERICAL METHOD}

\section{PHYSICAL MODEL AND MESH GENERATION.}

This paper selects the high-pressure stage positive bending twisted blade of some domestic turbine as the research object, and basic geometric parameters of the blade are as shown in Table 1.

For the geometrical model and mesh generation of cascade, see Fig. 1. All of the meshes in the blade passage adopt structured hexahedral mesh; the blade tip clearance adopts the H-type mesh, and is locally ciphered, to ensure enough accuracy of the flow field. After the mesh independence verification, the total number of divided meshes is about 630,000 , among which, the meshes numbers of the axial, circumference and spanwise of the blade passage are respectively $112 * 50 * 68$, and the meshes number of the labyrinth clearance is $166^{\star} 159{ }^{\star} 10$.

Tab. 1.Basic Geometric Parameters of Moving Blade

\begin{tabular}{ll}
\hline Parameters & Numerical Value \\
\hline Length of blade chord/mm & 35.04 \\
Length of axial chord/mm & 24.81 \\
Blade height/mm & 70.8 \\
Pitch/mm & 28.2 \\
Leading edge radius $/ \mathrm{mm}$ & 3.305 \\
Trailing edge radius $/ \mathrm{mm}$ & 0.154 \\
Blade setting angle $/{ }^{\circ}$ & 48.69 \\
Entrance geometrical angle $/{ }^{\circ}$ & 42 \\
Angle of attack $/{ }^{\circ}$ & 0 \\
\hline
\end{tabular}

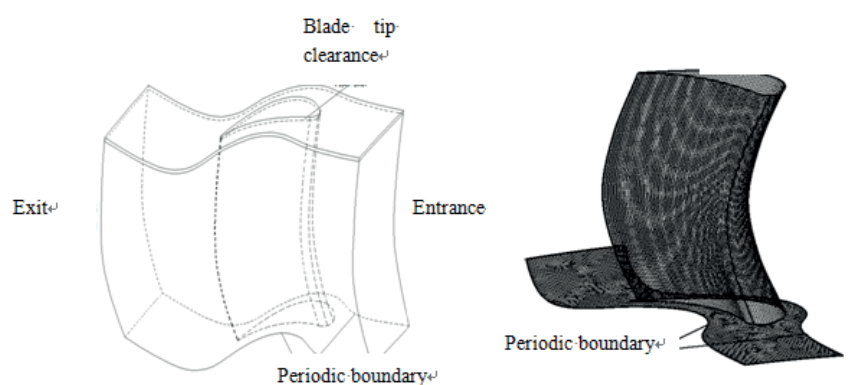

Fig. 1. Geometrical Model and Mesh Generation of Cascade Passage

1.2 Numerical Methods and Boundary Conditions

Using the Fluent software to solve the three dimensional steady viscous Reynolds average equations, and using finite volume method based on unit center solves the governing equations. Turbulence model selects ${ }^{\mathrm{k}-\omega}$ SST model, and nearby the wall adopts the wall-function method; Use SIMPLEC algorithm to solve the pressure-speed coupling and the discrete set of the convective item of the control equation is the second order upwind scheme. The revolving speed of moving blades is 3,000 RPM. The inlet boundary condition is the pressure entrance, with the pressure of $11.0495 \mathrm{MPa}$, and the temperature of $750 \mathrm{~K}$; the outlet boundary condition is the pressure exit, with pressure of $10.697 \mathrm{MPa}$, and the temperature of $734 \mathrm{~K}$. The fluid of the computational domain chooses overheating state seam. In the process of calculation, through the observation of each equation convergence condition, properly adjust relaxation factors, to speed up to calculate the convergence. The residual error to reach convergence of continuity equations, velocity equations, turbulence energy and turbulence dissipation rate is 10-3, and that of energy equations is 10-7.

\section{COMPUTATION RESULTS AND ANALYSIS}

\section{DISTRIBUTION OF STATIC PRESSURE ALONG BLADE PROFILE IN DIFFERENT BLADE HEIGHT SECTIONS}

Fig. 2 is distribution of the static pressure of conventional twisted blades and positive bending twisted blades along blade profile in each blade height section.

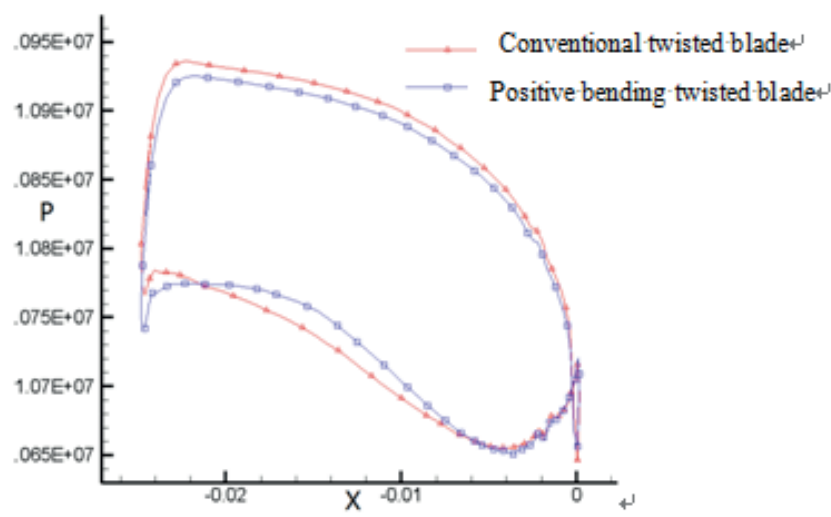

(a) Distribution of Static Pressure of 10\% of the Blade Height Section 


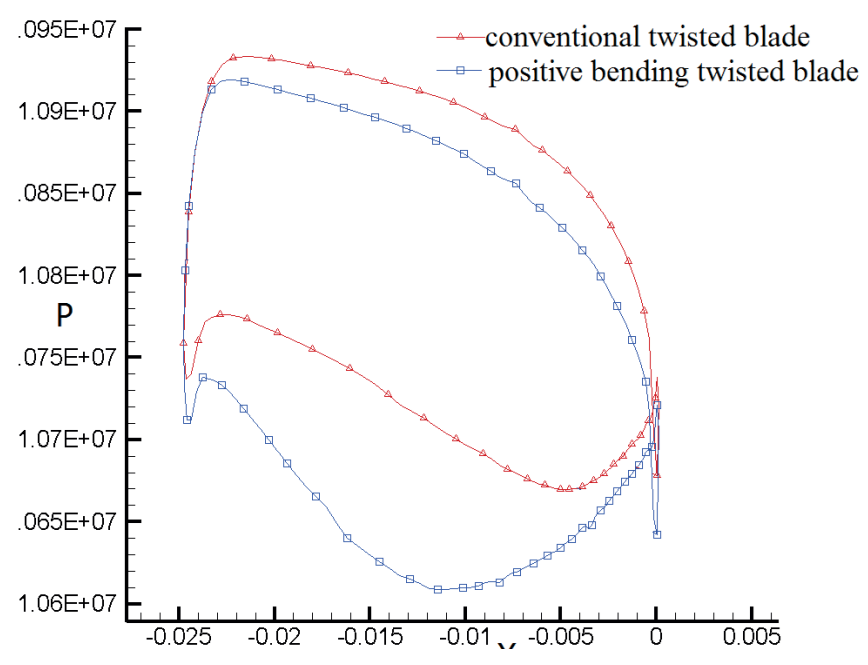

(b) Distribution of Static Pressure of 50\% of the Blade Height Section

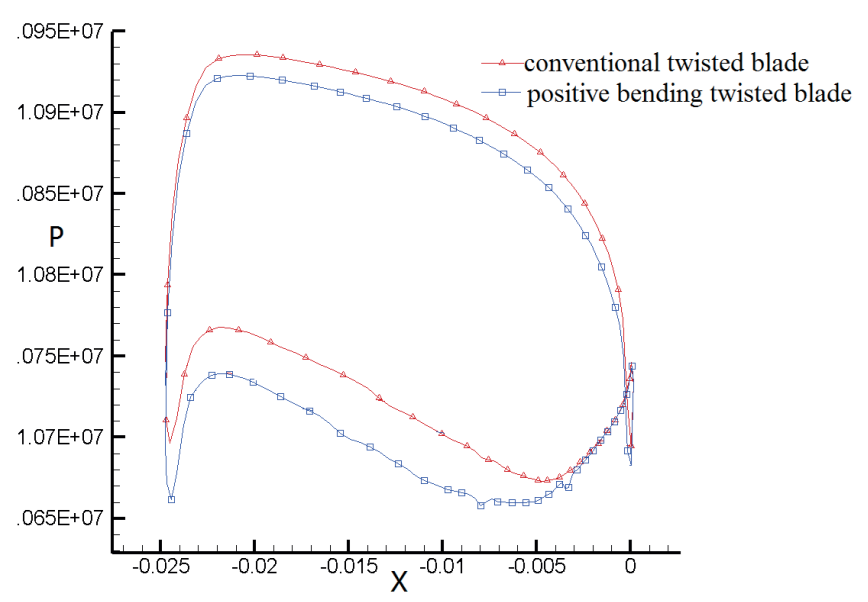

(c) Distribution of Static Pressure of $90 \%$ of the Blade Height Section

Fig. 2. Distribution of Static Pressure along Blade Profile in Different Blade Height Sections

As can be seen from Fig. 2, after the steam flow bypassed leading edge point of the blade, the pressure of the pressure surface all gradually reduce along the blade profile; the pressure of suction surface also gradually decreases along the blade profile within the first $80 \%$ range of the cascade axial, but within $20 \%$ range of the cascade back end, an increase sign appears. This is because in this area, the passage secondary vortex of the cascade flow path under the action of the transverse pressure gradient gathers in the suction surface, and the boundary layer of the suction surface here receives disturbance of the secondary vortex, starts to break away from the suction surface, and mixes together with the passage vortex, which makes the velocity of the steam flow decrease and the pressure increase therewith.

Compared to the Fig. (a), (b), (c) in Fig. 2, we can also see that, relative to the conventional twisted blade, although the change of the transverse pressure gradient of the pressure surface and suction surface of the positive bending twisted blade between the root and the tip of the blade is not big, it is quite obvious in the middle of the blade. This is due to after the twisted blade bent positively, " $\mathrm{C}$ " type pressure distribution is formed both on the suction surface and the pressure surface of the blade, which makes transverse pressure gradient in the middle of the blade increase, the diffusion section of the middle of the suction surface increase, and the transverse pressure gradient at the end of the blade reduce, and the diffusion section at the end of the suction surface reduce.

The end secondary flow of the blade is mainly determined by the transverse pressure gradient, so as long as the transverse pressure gradient decreases, the transverse secondary flow of the two ends can be weakened, and then interaction between the secondary flow and the mainstream cascade passage weakens, and the mixing loss caused by the secondary flow reduces.

\section{DISTRIBUTION OF STATIC PRESSURE COEFFICIENT IN THE BLADE TIP CLEARANCE OF POSITIVE BENDING TWISTED BLADES}

Fig. 3 is the contour line distribution diagram of static pressure coefficient of positive bending twisted blade clearance section under different blade tip clearances.

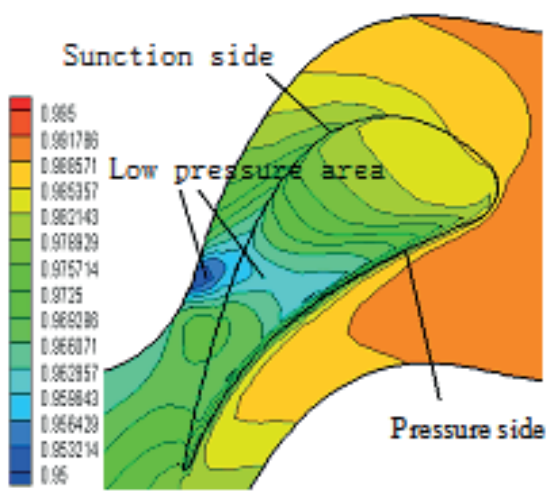

(a) $\tau=1 \mathrm{~mm}$

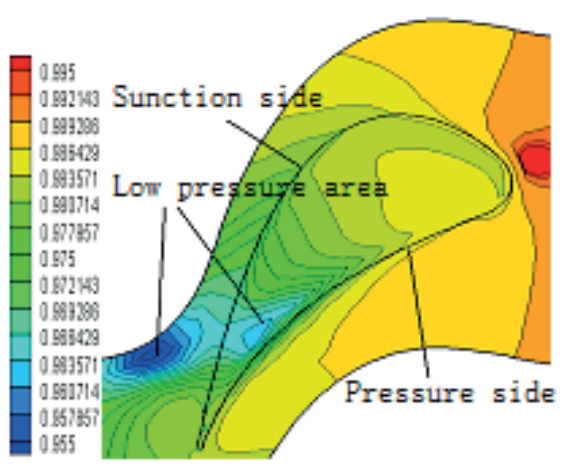

(b) $\tau=2 \mathrm{~mm}$

Fig. 3. Distribution Diagram of Static Pressure Coefficient in Blade Tip Clearance

As shown in Fig. 3, due to a larger transverse pressure gradient existing between the pressure side and suction side of the blade, part of steams in the passage will flow from the side 
of the pressure side of the tip clearance to the side of suction side under the action of the transverse pressure gradient, forming the leakage flow of blade tip clearance. With the development of the flow, the leakage flow will eventually flow from the suction side of the blade into the adjacent cascade passage, and interblend the mainstream fluid within the passage, and form complex leakage vortex of tip clearance, resulting in the flow loss.

Compared to (a) and (b) in Fig. 3, you can see that with the increase of the tip clearance, the transverse pressure gradient between the pressure side and suction side of the clearance is increasing, which causes the increase of the blade tip clearance leakage rate. In addition, with the increase of the blade tip clearance, the leakage vortex of the blade tip clearance gradually shifts to the downstream of the cascade and gradually gets away from the suction side of the blade. This is because the larger the blade tip clearance, the greater the leakage flow velocity, and the higher the ability to push the mainstream in the cascade passage, at this moment, the leakage loss and the mixing loss caused by the leakage flow are more.

\section{DISTRIBUTION OF STATIC PRESSURE OF THE SUCTION SURFACE OF POSITIVE BENDING TWISTED BLADES}

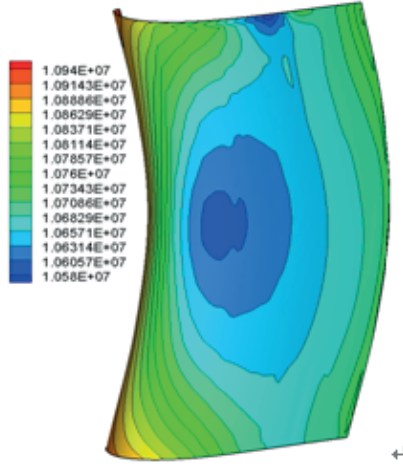

(a) $\tau=1 \mathrm{~mm}$

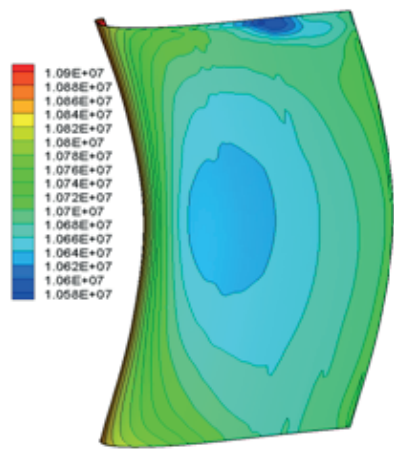

(b) $\tau=2 \mathrm{~mm}$
Fig. 4 Distribution Diagram of Static Pressure of the Suction Side

Fig. 4 is the contour line distribution diagram of static pressure of the positive bending twisted blade tip clearance suction surface under different blade tip clearances. From Fig. 4 , we can see that with the increase of the blade tip clearance, the scopes of influence of the clearance leakage flow low pressure area in the axial direction and spanwise of the blade somewhat increase. The increase of the low pressure area scope of influence, explains somewhat increase of the loss of pressure of steams, while the increase of leakage loss is just accompanied by the increase of the loss of pressure, thus it can again explain that, the clearance leakage loss caused by the leakage flow increases with the increase of the blade tip clearance.

In addition, the increase of the blade tip clearance, to some extent, weakens the " $C$ " type pressure gradient formed on the suction surface by the positive bending twisted blade. The decrease of "C" type pressure gradient will make reducing of the power transmitted by low steam energy at both ends of the blade to the central section, so, the low energy steam will be accumulated in the two ends of the blade, which will result in thickness of the boundary layer at the blade end becoming larger, and the end loss can also increase accordingly.

\section{CONCLUSIONS}

(1) After the twisted blade positively bends, under the action of "C" type pressure distribution, the transverse pressure gradients at two end of the blade reduce, which helps to reduce the transverse secondary flow at the end, and weakens interaction between the secondary flow and cascade passage mainstream, and thus to reduce the mixing loss of the secondary flow and the passage mainstream.

(2) With the increase of the blade tip clearance, the tip clearance leakage vortex of the positive bending twisted blade gradually transfers to the downstream of the cascade and gradually away from the blade suction surface, the scope of influence in the cascade passage gradually increases, and the corresponding leakage loss and the mixing loss also gradually increase.

(3) The increase of the blade tip clearance weakens "C" type pressure gradient on the suction surface of the positive bending twisted blade, makes the boundary layer thickness at both ends of the blades increase, and causes the increase of the loss of the blade end.

\section{ACKNOWLEDGEMENTS}

This work was financially supported by Department of Jilin Province Natural Science Foundation of China(20140101064JC)

\section{REFERENCES}

1. H.S.Chen, S. Kang and C.Q. Tan, "Experiment Research on the Influence of Positive bending of the Blade on the Aerodynamic Characteristics of Penetrated Calm Cascade and Blade", Engineering Thermophysics Newspaper, no. 2, pp. 179-182, 2002,.

2. G Pullan and N.W Harvey, "The influence of sweep on axial flow turbine aerodynamics in the endwall region", Journal of Turbomachinery, no.130, 2008,.

3. M. H. Vand and S. Wang, "Numerical Study of the Effects of Bowed Blades on Aerodynamic Characteristics in a High-pressure Turbine", American Society of Mechanical Engineers, pp. 487-496, 2005,

4. B.T. An, W.J. Han and Z.Q Wang, "Experience Research on Mechanism of Twisted Blades Reducing Losses", Thermal Energy Power Engineering, no. 15, pp. 498-501, 2000,.

5. Z.M. Feng, W.A. Hang and J.J. Zhong, "Experimental Study on Post-load Positive Bending Cascade Aerodynamic Performance”, Experimental Fluid Mechanics, no. 23, pp. 
$56-59,2009$.

6. H.W. Lu, X.X. Kan and J.J. Zhong, etc. :Analysis of the Flow Structure in Positive Bending Compressor Stationary Guide Blade Passageway", Engineering Thermophysics Newspaper, no. 34, pp. 1828-1832, 2013,.

7. L. Chen, and J. Cheng, "Numerical Investigation on the Influence of Twisted Blades on Turbine Aerodynamic Performance under Stage Environmen", Aviation Dynamics Newspaper, no. 26, pp. 2765-2711, 2011,.

8. Atkins, RJ (Atkins, Rowland J.); Tidd, M (Tidd, Morgan); Ruffo, G (Ruffo, Gord). "Sturgeon Bank, Fraser River Delta, BC, Canada: 150 Years of Human Influences on Salt Marsh Sedimentation", Journal of Coastal Research, SI 75, pp. 790-794, 2016,

\title{
CONTACT WITH AUTHOR
}

\author{
Jianguo Jin \\ email: jinjianguo6503@163.com, \\ Zhanzhou Wang \\ wang_zhan_zhou@163.com, \\ Lihua Cao \\ email:984066765@qq.com \\ School of Energy and Power Engineering, \\ Northeast Dianli University, \\ Jilin, \\ China
}

